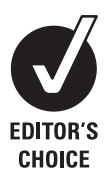

${ }^{1}$ Department of Medicine, division of Cardiovascular Diseases, Mayo Clinic, Rochester Minnesota, USA; ${ }^{2}$ Department of Radiology, Mayo Clinic, Rochester Minnesota, USA

Correspondence to:

Dr Ronen Rubinshtein, Division of Cardiovascular Diseases, MB 4-506, 200 First Street SW,

Rochester, MN 55905, USA rubinshtein.ronen@mayo.edu

Accepted 27 January 2009

\title{
Detection of myocardial infarction by dual-source coronary computed tomography angiography using quantitated myocardial scintigraphy as the reference standard
}

\author{
R Rubinshtein, ${ }^{1}$ T D Miller, ${ }^{1}$ E E Williamson, ${ }^{2} \mathrm{~J} \mathrm{Kirsch,}^{2}$ R J Gibbons, ${ }^{1}$ A N Primak, ${ }^{2}$ \\ C H McCollough, ${ }^{2}$ P A Araoz ${ }^{2}$
}

\begin{abstract}
Background: Dual-source coronary computed tomography angiography (DS-CTA) has the potential to assess both coronary anatomy and myocardial perfusion. We studied the ability of DS-CTA to detect myocardial infarction (MI) compared to a reference standard of technetium $T c^{99} \mathrm{~m}$ sestamibi single photon emission computed tomography (SPECT).
\end{abstract}

Methods: 122 patients with suspected or known coronary artery disease (age 60 (SD 11) years, 36\% females) were evaluated by both DS-CTA and SPECT. SPECT-MI size was quantitated using a threshold value of $60 \%$ of peak counts on the resting images. MI on DS-CTA was defined as transmural or subendocardial hypoenhancement ( $<50 \%$ of surrounding myocardium), which persisted in both diastolic and systolic reconstructions and was concordant with a coronary artery territory. The performance of DS-CTA to detect SPECT-MI was determined in a blinded, vessel-based analysis.

Results: 366 vessel territories were analysed (122 patients $\times 3$ ). SPECT revealed 20 vessel territories with MI (involving 17 patients). 15/20 (75\%) of these vessel territories were also detected by DS-CTA. An additional seven Mls were detected by DS CTA only (considered as false positive). Thus, the sensitivity of DS-CTA for detection of SPECT-MI was 75\% (95\% Cl 56\% to 94\%), specificity $98 \%(97 \%$ to $100 \%)$, positive predictive value $68 \%(49 \%$ to $88 \%$ ) and negative predictive value $99 \%$ (97\% to 100\%). DS-CTA detected 10/11 (91\%) larger Mls (involving $>5 \%$ of left ventricular (LV) mass by SPECT). For the 15 concordant Mls (in both SPECT and DS-CTA) the mean difference in $\mathrm{MI}$ size between modalities was $0.5 \%$ (4.6\%) of LV mass ( $95 \% \mathrm{Cl}-8.6 \%$ to $9.5 \%$ ).

Conclusions: DS-CTA myocardial perfusion imaging showed moderate sensitivity and positive predictive value but high specificity and negative predictive value for detection of SPECT-MI. Most large infarcts (>5\% of LV mass) were detected by DS-CTA. When MI was detected by both modalities, there was a good correlation between infarct sizes quantitated by DS-CTA vs SPECT.

Multi-detector computed tomography (MDCT) has become an important modality for evaluation of coronary anatomy in various patient populations and especially in the evaluation of patients with chest pain. ${ }^{1-3}$ While the primary and most common indication for coronary computed tomography (CT) angiography is detection of coronary artery stenosis, additional important information may be acquired during cardiac evaluation with
MDCT ${ }^{4}$ One additional piece of information could be myocardial viability. Myocardial hypoenhancement during first-pass MDCT is thought to represent myocardial infarction (MI). This finding has been described in both humans and animal models. ${ }^{5-10}$ The pathophysiology of myocardial hypoenhancement by CT is similar to that of other imaging modalities, caused by reduced microvascular blood flow in the infarcted area leading to decreased concentration of contrast (iodine or gadolinium) or radioactive tracers. ${ }^{5}$

Single photon emission computed tomography (SPECT) is the most extensively validated methodology for quantification of infarct size. ${ }^{11}$ Only limited data are available regarding the ability of first-pass MDCT to diagnose MI compared to SPECT. ${ }^{12}$ The purpose of our study was to evaluate the diagnostic accuracy of first-pass coronary CT angiography using a modern dual-source scanner to diagnose MI using SPECT as the reference standard.

\section{METHODS}

\section{Patient selection}

This study was a retrospective analysis of data in all patients who underwent both SPECT and dualsource coronary CT angiography (DS-CTA) for a clinical indication within 120 days of each other, and identified by cross-matching electronic SPECT and DS-CTA databases. During a 19-month period, 5082 patients underwent technetium $\mathrm{Tc}^{99} \mathrm{~m}$ sestamibi scans and 344 underwent coronary DS-CTA, of whom 149 potentially eligible patients were identified. Six patients did not grant research authorisation and 21 patients were excluded because of an acute coronary syndrome between examinations. The final study group consisted of 122 patients. The time between examinations was 7 (45) days, and $\leqslant 7$ days in 77 patients.

\section{Dual-source CT angiography}

Coronary CT angiography scans were performed on a dual-source CT scanner (Definition DS, Siemens Healthcare, Erlangen, Germany). Oral $\beta$ blocker (metoprolol 50-100 mg) was given before examination only if heart rate was $>80$ beats per minutes (bpm). Patients received sublingual nitroglycerin (unless contraindicated). A contrast enhanced scan was then performed with a bolus of 111 (19) $\mathrm{ml}$ contrast medium (Omnipaque 350 or Visipaque 320) injected ("Care Bolus" protocol) 
into an antecubital vein at a flow rate of $4-5 \mathrm{ml} / \mathrm{s}$. Scanning was performed at $120 \mathrm{kVp}$, tube current-time product: $380 \mathrm{mAs} /$ rotation, using a slice collimation of $2 \times 32 \times 0.6 \mathrm{~mm}$, a 0.33 -second gantry rotation time and heart-rate dependent, adaptive pitch. ECG-based tube current modulation was used in all patients (with a full dose window of $60-70 \%$ of the cardiac cycle in patients with heart rate $<65 \mathrm{bpm}$ and $40-70 \%$ in patients with higher heart rate or post bypass surgery). Dose length product (DLP) was calculated by multiplying the volume CT dose index $\left(\mathrm{CTDI}_{\mathrm{vol}}\right)$ and scan length. DLP for coronary CT angiography was 1128 (423) mGy $\times$ cm (range 449-2452), depending on patient size, scan length, heart rate and pitch.

DS-CTA scans were reconstructed with a slice thickness of $0.75 \mathrm{~mm}$ and a $0.4-\mathrm{mm}$ increment using an edge-preserving, noise-reducing reconstruction kernel (B26). ${ }^{13}$ Two experienced observers (blinded to SPECT results) assessed the presence of myocardial infarction by consensus. Myocardial infarction on DS-CTA was defined as subendocardial or transmural hypoenhancement $(<50 \%$ attenuation of the surrounding myocardium), which persisted in both systolic and diastolic reconstructions and was concordant with a coronary territory. Evaluation of reconstructed images for hypoenhancement was performed beginning with a narrow window/level (centre 100 $\mathrm{HU}$, width $200 \mathrm{HU}$ ) with manual adjustment. In patients in whom MI was diagnosed by both DS-CTA and SPECT, quantification of MI size was manually performed ("Argus" CT application, Siemens Healthcare). Initially, the epicardial border and endocardial borders were marked from the enddiastolic phase (to calculate left ventricular (LV) end-diastolic mass) and then the infarct itself was traced to quantify MI size (expressed as percentage of LV mass).

\section{Single photon emission computed tomography}

The stress SPECT methods have been previously described. ${ }^{14} 15$ Rest imaging was performed before stress imaging. Following injection of $8-12 \mathrm{mCi}$ of technetium $\mathrm{Tc}^{99} \mathrm{~m}$ sestamibi, rest SPECT imaging was performed over a $180^{\circ}$ arc with the "stepand-shoot" method. Filtered back projection was performed with a Ramp-Hanning filter. As previously reported, ${ }^{16}$ infarct quantification was performed on the rest SPECT image utilising a threshold of $<60 \%$ of peak counts and five short-axis slices of the LV extending from apex to base. Infarct size was expressed as a percentage of the LV mass. Infarct location was determined by a blinded experienced observer according to vessel territory utilising the 17 segment model. ${ }^{17}$

\section{Statistical analysis}

Sensitivity, specificity, positive and negative predictive values of DS-CTA to detect MI were analysed according to vessel territory and compared to SPECT as the gold standard. MI detected by DS-CTA only was considered a false positive. MI detected by SPECT only was considered a false negative. BlandAltman analysis was performed to correlate MI size when present in both modalities and paired t test was used to evaluate potential bias between measurements. Statistical analysis was performed using Statistix 8 software (Analytical Software, Tallahassee, FL, USA). A p value of $<0.05$ was considered significant. The study was approved by the institutional review board with waiver of informed consent.

\section{RESULTS}

SPECT and DS-CTA scans from 122 patients were analysed. Baseline characteristics are shown in table 1.
SPECT diagnosed MI in 20 vessel territories in 17 patients. Fifteen of these patients (88\%) had a previous history of myocardial infarction or coronary revascularisation. MI was present in $14 \%(17 / 122)$ of the patients and $5.5 \%(20 / 366)$ of vessel territories. DS-CTA detected 15 of the 20 infarcts diagnosed by SPECT.

Seven infarcts were diagnosed by DS-CTA only (false positives). In all, 339 vessel territories were negative for infarcts by both rest SPECT and DS-CTA (true negatives). Five infarcts were diagnosed by SPECT only (false negatives). Thus, the sensitivity of DS-CTA for detection of SPECT-MI (per vessel territory) in our cohort was $75 \%$ (95\% CI $56 \%$ to $94 \%$ ), specificity $98 \%$ (97\% to $100 \%$ ), positive predictive value $68 \%$ (49\% to $88 \%$ ) and negative predictive value $99 \%$ (97\% to $100 \%)$ (fig 1).

Performance characteristics of DS-CTA for detection of SPECT-MI on a patient-based analysis were quite similar to the vessel based analysis. Of the $17 / 122$ patients in whom MI was diagnosed by SPECT; DS-CTA diagnosed MI in 13 and six patients had false-positive DS-CTA results. Thus, on a patientbased analysis the sensitivity of DS-CTA to detect SPECT-MI was $77 \%$ (95\% CI $56 \%$ to $97 \%$ ), specificity $94 \%$ ( $90 \%$ to $99 \%$ ), positive predictive value $68 \%$ (47\% to $89 \%$ ) and the negative predictive value was $96 \%$ ( $92 \%$ to $100 \%$ ) (fig 1).

Interestingly, comparison between vessel-based results and patients' baseline characteristics showed that six of the seven false-positive infarcts by DS-CTA were detected in patients with known coronary artery disease and a history of revascularisation. Six of the seven "false-positive" DS-CTA infarcts were also sub-endocardial in distribution (example in fig 2), and those six were relatively small ( $<5 \%$ of LV mass).

Among the five infarcts demonstrated by SPECT but not diagnosed by DS-CTA (false negative), three were located in the inferior wall and two in the anterior wall, and only one infarct was $>5 \%$ of LV mass; $10 / 11$ (91\%) of SPECT MIs larger than $5 \%$ were also recognised by DS-CTA.

For the 15 MIs detected by both SPECT and DS-CTA (diagnosed in 13 patients), the mean MI size by DS-CTA was $11 \%(7.6 \%)$, and mean MI size by SPECT was $11.5 \%$ (8.1\%) of LV mass. Bland-Altman analysis showed that the difference in measurements between modalities was $0.5 \%$ (4.6\%) of LV mass

Table 1 Baseline characteristics of 122 patients undergoing dual-source coronary CT angiography and SPECT

\begin{tabular}{lc}
\hline Age (years) (SD) & $60(11)$ \\
Females & $44(36 \%)$ \\
Presenting symptoms: & $13(11 \%)$ \\
$\quad$ Typical angina & $43(35 \%)$ \\
Atypical angina & $4(3 \%)$ \\
Non-cardiac chest pain & $14(12 \%)$ \\
Dyspnoea & $48(39 \%)$ \\
Other/asymptomatic & $45(36 \%)$ \\
Known coronary artery disease & $8(7 \%)$ \\
Documented previous myocardial infarction & $15(12 \%)$ \\
Previous percutaneous coronary intervention & $16(13 \%)$ \\
Previous bypass surgery & $20(16 \%)$ \\
Diabetes mellitus & $59(48 \%)$ \\
Systemic hypertension & $81(66 \%)$ \\
Hyperlipidaemia & $13(10 \%)$ \\
Smoker (current or previous) & $20(16 \%)$ \\
Family history of coronary artery disease & $29.6(4.7)$ (range \\
Body mass index (kg/m²) (mean (SD)) & $18.3-41.2)$ \\
\hline
\end{tabular}




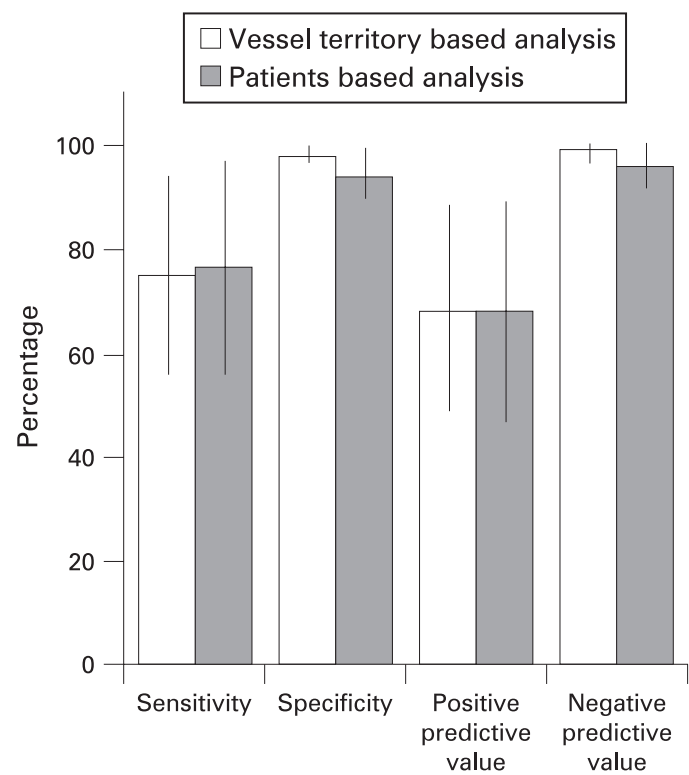

Figure 1 Performance characteristics of DS-CTA to detect SPECT-MI in 122 patients (histogram includes $95 \%$ confidence intervals).

(95\% CI $-8.6 \%$ to $9.5 \%$ ), and bias between measurements was not significant ( $p=0.72$, using paired t test) (fig 3 ).

\section{DISCUSSION}

The main finding of our study is that (first pass) DS-CTA performed originally for evaluation of the coronary vessels was capable of diagnosing $75 \%$ of myocardial infarctions using SPECT as the reference standard. This was achieved by using initially a narrow window/level to detect myocardial hypoenhancement, with subsequent manual adjustment. Moreover, our results demonstrate the ability of DS-CTA to diagnose MI among a large cohort with a low prevalence of MI.

In addition, in patients where MI was diagnosed by both modalities, infarct size measured by dedicated CT applications was significantly correlated with SPECT infarct size, and mean difference in MI size between modalities was not much different from the interobserver variability reported for SPECT. ${ }^{18}$
The ability of DS-CTA to detect myocardial infarction, in addition to assessment of coronary anatomy during coronary CT angiography, can add important clinical information. The main purpose of coronary DS-CTA is to diagnose coronary artery plaque and narrowing in patients at low to intermediate risk. ${ }^{1}$ With current techniques, DS-CTA is probably not indicated for the sole purpose of ruling out MI. However, the information needed to diagnose $\mathrm{MI}$ is already available from the coronary CT scan and can be used in most patients. The detection of infarcted myocardium can add support to the diagnoses of significant coronary stenosis by CTA, and may also have prognostic implications. ${ }^{611}$ Our study adds to the findings of a recent study that included patients with known healed MIs and reported $100 \%$ sensitivity of hypoenhancement by 64 -slice CT to diagnose SPECT MI. ${ }^{12}$ We report lower sensitivity (75\%), which possibly is related to stricter criteria for diagnosing MI by CT $(<50 \%$ attenuation of the surrounding myocardium, and requirement for presence in both systolic and diastolic reconstructions). The ability of CT to diagnose $\mathrm{MI}$ and to predict functional recovery following acute MI has already been demonstrated, especially with delayed enhancement acquisitions. ${ }^{6}$ Delayed MDCT scans were also shown to correlate well with magnetic resonance imaging for evaluation of MI size, transmurality and degree of microvascular obstruction. ${ }^{19}$ However, performing delayed scans, which requires additional scanner time and increases patient radiation exposure may not be a practical option in some patients or institutions.

The CT scans that were "false positive" (using SPECT was our reference standard) may possibly have been "false negative" SPECT studies (an example is shown in fig 2). The extensive experience with SPECT has demonstrated that SPECT has suboptimal sensitivity for smaller infarcts ( $<3 \%$ of LV mass) and subendocardial infarcts. ${ }^{11} 2021$ Similar to magnetic resonance imaging, CT may be able to detect sub-endocardial MI that is not detectable by SPECT, but this issue requires further investigation.

Furthermore, while the incremental benefit of DS-CTA over single-source CT in evaluating the myocardium for ischaemia and infarction may be small. The introduction of dual energy protocols to DS-CTA in the future may define changes in myocardial tissue perfusion even better. ${ }^{22}$

Another possible limitation of our results is the limited number of vessel areas with MI among our patients. This may
Figure 2 Subendocardial anterior myocardial infarction demonstrated by DS-CTA (A, white arrow), but not by SPECT (B). The electrocardiogram of a 58-year-old patient with known previous $\mathrm{MI}$ and a history of angioplasty to the left anterior descending coronary artery is shown in (C). Diffuse atherosclerosis in the proximal left anterior descending coronary artery is shown in (D). Although considered as false-positive DS-CTA for our analysis, may indeed be a falsenegative SPECT.
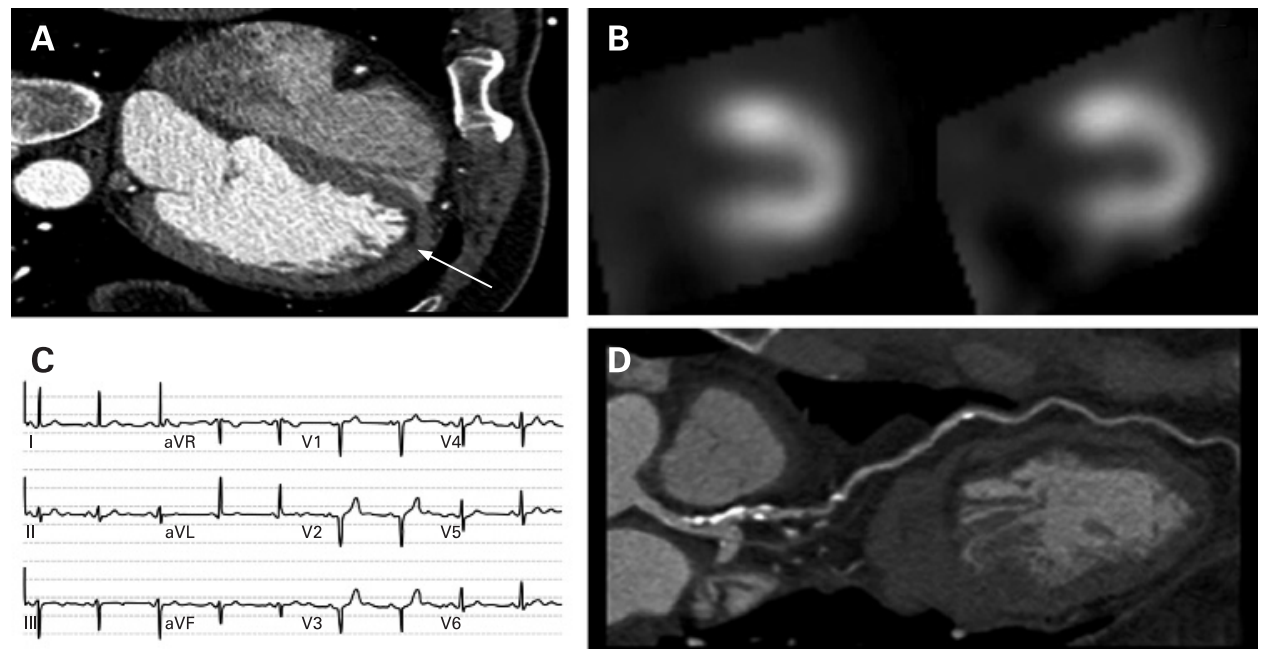


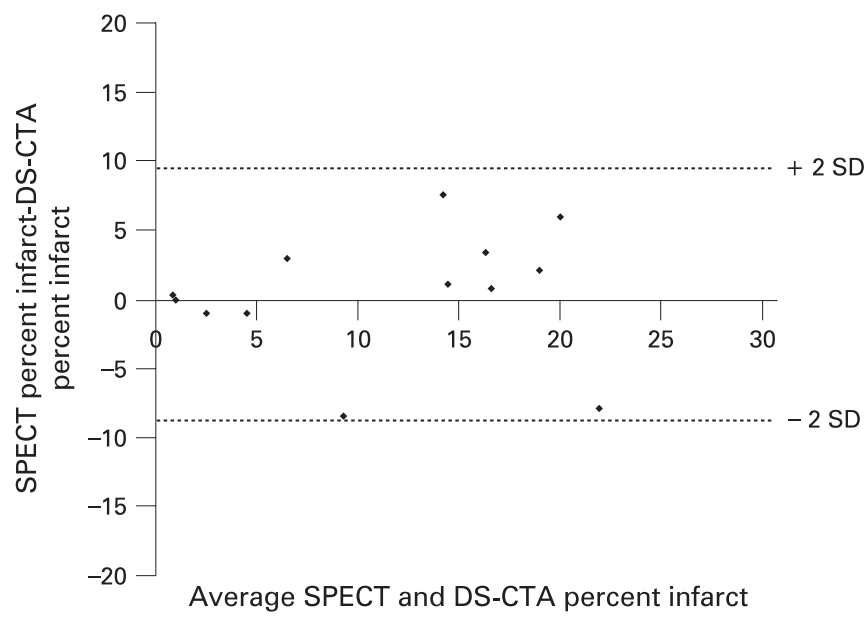

Figure 3 Bland-Altman analysis comparing infarct size between dualsource coronary computed tomography angiography (DS-CTA) and single photon emission computed tomography (SPECT) in 13 patients (15 infarcts) in whom both examinations were positive for myocardial infarction (MI).

have increased the negative predictive value but should not have an effect on sensitivity and specificity.

The role of DS-CTA for evaluation of patients with known coronary artery disease and previous revascularisation versus SPECT has not been established. Radiation exposure is a matter of concern, ${ }^{23}$ especially if utilising both modalities and the advantage of anatomical approach (CT) versus a physiological approach (SPECT) in such patients deserves further study.

While our cohort consisted of patients who underwent both examinations for a clinical indication, we do not recommend routine evaluation of symptomatic patients by both modalities, nor can we extrapolate results from this study to all patients with acute coronary syndromes (as acute coronary event between examinations was an exclusion criteria).

In conclusion, our study shows that first-pass DS-CTA is able to detect MI with a moderate sensitivity and high specificity when compared to SPECT. This methodology can provide clinically useful information without any additional radiation or scanning time.

Acknowledgements: The authors would like to thank Natalie N Braun for her valuable assistance.

Funding: TDM received research grants from Lantheus Medical Imaging and Molecular Insight Pharmaceuticals and has acted as a consultant to TherOx, Inc and The Medicines Company. RJG received research grants from Radiant Medical, KAI Pharmaceuticals, TargeGen, TherOx and King Pharmaceuticals, and has acted as a consultant for Hawaii Biotech, Cardiovascular Clinical studies (WOMEN study), Consumers Union and the TIMI 37A study. EEW received research grants from Siemens Medical Solutions and Bayer Health care and participated in expert panels for both. ANP is partially supported by a research grant from Siemens Medical Solutions. $\mathrm{CHMcC}$ received research grants from Siemens Medical Solutions, Bayer Health care, and RTI electronics.

\section{Competing interests: None.}

The authors had full access to data and take full responsibility for the integrity of the data. All authors have read and agree to the manuscript as written.

\section{REFERENCES}

1. Schroeder S, Achenbach S, Bengel F, et al, European Society of Cardiology; European Council of Nuclear Cardiology. Cardiac computed tomography: indications, applications, limitations, and training requirements: report of a Writing Group deployed by the Working Group Nuclear Cardiology and Cardiac CT of the European Society of Cardiology and the European Council of Nuclear Cardiology. Eur Heart $J$ 2008;29:531-56.

2. Rubinshtein R, Halon DA, Gaspar T, et al. Usefulness of 64-slice multidetector computed tomography in diagnostic triage of patients with chest pain and negative or nondiagnostic exercise treadmill test result. Am J Cardiol 2007;99:925-9.

3. Rubinshtein R, Halon DA, Gaspar T, et al. Usefulness of 64-slice cardiac computed tomographic angiography for diagnosing acute coronary syndromes and predicting clinical outcome in emergency department patients with chest pain of uncertain origin. Circulation 2007;115:1762-8.

4. Schuijf JD, Bax JJ. CT angiography: an alternative to nuclear perfusion imaging? Heart 2008:94:255-7.

5. Lessick J, Ghersin E, Dragu R, et al. Diagnostic accuracy of myocardial hypoenhancement on multidetector computed tomography in identifying myocardial infarction in patients admitted with acute chest pain syndrome. J Comput Assist Tomogr 2007;31:780-8.

6. Lessick J, Dragu R, Mutlak D, et al. Is functional improvement after myocardial infarction predicted with myocardial enhancement patterns at multidetector CT? Radiology 2007;244:736-44.

7. Nikolaou K, Sanz J, Poon M, et al. Assessment of myocardial perfusion and viability from routine contrast-enhanced 16-detector row computed tomography of the heart: preliminary results. Eur Radiol 2005;15:864-71.

8. Park JM, Choe YH, Chang S, et al. Usefulness of multidetector-row CT in the evaluation of reperfused myocardial infarction in a rabbit model. Korean $J$ Radiol 2004:5:19-24.

9. Gerber BL, Belge B, Legros GJ, et al. Characterization of acute and chronic myocardial infarcts by multidetector computed tomography: comparison with contrast-enhanced magnetic resonance. Circulation 2006;113:823-33.

10. Lardo AC, Cordeiro MA, Silva C, et al. Contrast-enhanced multidetector computed tomography viability imaging after myocardial infarction: characterization of myocyte death, microvascular obstruction, and chronic scar. Circulation 2006;113:394-404.

11. Gibbons RJ, Valeti US, Araoz PA, et al. The quantification of infarct size. J Am Coll Cardiol 2004:44:1533-42.

12. Henneman MM, Schuijf JD, Dibbets-Schneider P, et al. Comparison of multislice computed tomography to gated single-photon emission computed tomography for imaging of healed myocardial infarcts. Am J Cardiol 2008;101:144-8.

13. McCollough CH, Primak AN, Saba 0, et al. Dose performance of a 64-channel dualsource CT scanner. Radiology 2007:243:775-84.

14. Christian TF, Miller TD, Bailey KR, et al. Noninvasive identification of severe coronary artery disease using exercise tomographic thallium-201 imaging. Am J Cardiol 1992; 70:14-20.

15. Johnston DL, Daley JR, Hodge DO, et al. Hemodynamic responses and adverse effects associated with adenosine and dipyridamole pharmacologic stress testing: a comparison in 2,000 patients. Mayo Clin Proc 1995;70:331-6.

16. Gibbons RJ, Miller TD, Christian TF. Infarct size measured by single photon emission computed tomographic imaging with $(99 \mathrm{~m}) \mathrm{Tc}$-sestamibi: a measure of the efficacy of therapy in acute myocardial infarction. Circulation 2000;101:101-8.

17. Berman DS, Abidov A, Kang $X$, et al. Prognostic validation of a 17-segment score derived from a 20-segment score for myocardial perfusion SPECT interpretation. J Nucl Cardiol 2004;11:414-23.

18. Gibbons RJ, Verani MS, Behrenbeck T, et al. Feasibility of tomographic 99mTchexakis-2-methoxy-2-methylpropyl-isonitrile imaging for the assessment of myocardial area at risk and the effect of treatment in acute myocardial infarction. Circulation 1989:80:1277-86

19. Brodoefel H, Klumpp B, Reimann A, et al. Sixty-four-MSCT in the characterization of porcine acute and subacute myocardial infarction: determination of transmurality in comparison to magnetic resonance imaging and histopathology. Eur J Radiol 2007;62:235-46.

20. Wagner A, Mahrholdt H, Holly TA, et al. Contrast-enhanced MRI and routine single photon emission computed tomography (SPECT) perfusion imaging for detection of subendocardial myocardial infarcts: an imaging study. Lancet 2003;361:374-9.

21. Miller TD, Christian TF, Hopfenspirger MR, et al. Infarct size after acute myocardial infarction measured by quantitative tomographic $99 \mathrm{mTc}$ sestamibi imaging predicts subsequent mortality. Circulation 1995;92:334-41.

22. Schwarz F, Ruzsics B, Schoepf UJ, et al. Dual-energy CT of the heart-principles and protocols. Eur J Radiol 2008;68:423-33.

23. Brenner DJ, Hall EJ. Computed tomography - an increasing source of radiation exposure. N Engl J Med 2007;357:2277-84. 